\title{
Analisador de espessura retiniana (RTA) na avaliação de buraco macular
}

\author{
Retinal thickness analyzer(RTA) in evaluation of macularhole
}

\author{
Márcio Bittar Nehemy ${ }^{1}$ \\ Cláudia Borghi de Siqueira ${ }^{2}$ \\ Silvana Cecília Gomes Negrão ${ }^{2}$ \\ Márcio Zisman $^{3}$ \\ Elke Passos $^{4}$
}

\begin{tabular}{|l|}
\hline RESUMO \\
\hline Objetivo: Avaliar os resultados do Analisador de Espessura Retiniana \\
(RTA) em olhos com buraco macular e compará-los aos achados da \\
biomicroscopia do segmento posterior, retinografia e fluoresceinografia. \\
Métodos: Foram estudados por meio do analisador de espessura retiniana, \\
biomicroscopia do segmento posterior, retinografia e fluoresceinografia, \\
dez olhos de seis pacientes com diagnóstico de buraco macular. Destes, \\
8 foram examinados antes de qualquer tratamento, e dois, após o tratamen- \\
to do buraco macular pela vitrectomia. Resultados: Em dois olhos com \\
diagnóstico de buraco macular pelo exame clínico e outros exames comple- \\
mentares, a opacidade dos meios impediu a análise pelo analisador de \\
espessura retiniana. O corte óptico do analisador de espessura retiniana \\
mostrou concordância com os achados clínicos e com os observados nos \\
oito olhos em que o exame foi possível. O corte topográfico nos dois olhos \\
que apresentavam buraco macular fechado pela cirurgia mostraram espes- \\
sura foveal média normal, e nos seis olhos com buraco macular não \\
operado, espessura foveal média aumentada. Conclusão: O corte óptico \\
do analisador de espessura retiniana evidenciou o buraco macular, forne- \\
ceu informações sobre sua largura e profundidade e comprovou o fecha- \\
mento do buraco após a vitrectomia. O corte topográfico do analisador de \\
espessura retiniana mostrou espessura foveal média normal nos dois \\
casos de fechamento do buraco macular e, espessura foveal média aumen- \\
tada em todos os seis olhos não operados. \\
\hline
\end{tabular}

Descritores: Analisador de espessura retiniana; Técnicas de diagnóstico oftalmológico; Perfurações retinianas
Trabalho realizado no Hospital São Geraldo da Universidade Federal de Minas Gerais (UFMG) e no Instituto da Visão.

${ }^{1}$ Chefe do Serviço de Cirurgia Vítrea do Hospital São Geraldo da Universidade Federal de Minas Gerais, Prof Adjunto da Faculdade de Medicina da Universidade Federal de Minas Gerais. Chefe do Departamento de Retina e Vítreo do Instituto da Visão (Belo Horizonte MG), Prof. Titular da Faculdade de Medicina da Universidade Federal de Minas Gerais.

${ }^{2}$ Ex-fellow do Departamento de Retina e Vítreo do Instituto da Visão.

${ }^{3}$ Oftalmologista dos Setores de Retina e Catarata da Santa Casa de Misericórdia de Pernambuco e Preceptor da Disciplina de Oftalmologia da Universidade Federal de Pernambuco.

${ }^{4}$ Doutor em Oftalmologia pela Universidade Federal de Minas Gerais. Pós-doutorado, Louisiana State University e Tulane University, USA (Bolsista do CNPq). Oftalmologista do Departamento de Retina e Vítreo do Instituto da Visão (Belo Horizonte-MG).

Endereço para correspondência: Márcio B. Nehemy. Rua dos Otoni, 881 - $13^{\circ}$ and. - Belo Horizonte (MG) CEP30150-270.E-mail: oftalmologia@instvisão.com.br

\section{INTRODUÇÃ̃O}

Buraco macular idiopático é uma entidade clínica bem definida, que é acompanhada de significativa baixa da acuidade visual (BAV), usualmente de 0,2 ou pior. Afeta predominantemente mulheres na sexta década e tem uma prevalência de aproximadamente $0,3 \%$ em indivíduos acima de 55 anos $^{(1-2)}$. A BAV deve-se provavelmente à deiscência de tecido neurossensorial na região central da mácula, causada por retração centrífuga dos fotorreceptores secundária à contração e retração do vítreo na região foveolar e parafoveolar, e ao efeito de uma pequena orla de fluído subretiniano que circunda o buraco e separa a retina sensorial do epitélio pigmentar da retina $(\mathrm{EPR})^{(3)}$. Gass $^{(4)}$ sugere que o buraco macular começa como um descolamento da retina foveolar. Outros autores sugerem que a formação de cistos intra-retinianos estão associados com a formação do buraco macular ${ }^{(5-9)}$. 
Classicamente, o buraco macular é diagnosticado e avaliado por meio de biomicroscopia do segmento posterior, retinografia e fluoresceinografia. Esses dois últimos exames não avaliam a espessura da retina, e a biomicroscopia do segmento posterior, embora seja muito importante, oferece apenas uma informação subjetiva da espessura retiniana.

Exames capazes de avaliar o perfil e a espessura da retina no pólo posterior podem ser extremamente úteis para a avaliação de olhos com maculopatias, ${ }^{(10)}$ incluindo o buraco macular.

Vários aparelhos têm sido desenvolvidos para melhor estudar essas patologias. O Analisador de Espessura Retiniana (Retinal Thickness Analyzer - RTA) é um novo instrumento aprimorado a partir dos conceitos da biomicroscopia a laser, com capacidade de fornecer cortes ópticos da espessura da retina no pólo posterior, de maneira quantitativa e não subjetiva. Esse aparelho permite, também, a obtenção de mapas tridimensionais da retina no pólo posterior e mapas topográficos bidimensionais. Todos essas representações podem ser impressas e/ou armazenadas em discos ópticos para consultas posteriores $^{(11)}$.

Embora estudos prévios sugiram que o RTA possa auxiliar o diagnóstico e o acompanhamento da evolução do buraco macular, a literatura específica ainda é escassa, não existindo qualquer relato a esse respeito na literatura nacional. $O$ objetivo deste trabalho é avaliar os achados do RTA e compará-los aos achados da biomicroscopia do segmento posterior, da retinografia e da fluoresceinografia em pacientes com buraco macular.

\section{MÉTODOS}

\section{Pacientes}

Foram incluídos neste estudo seis pacientes consecutivos (10 olhos) com diagnóstico clínico de buraco macular - dos quais quatro apresentavam o quadro bilateralmente - que procuraram o Instituto da Visão no período de maio a agosto de 1998. Os pacientes deste estudo foram informados sobre todos os exames a que se submeteriam e concordaram com a sua implementação, assinando um termo de consentimento antes de todos os procedimentos. Em dois olhos com buraco macular o exame foi realizado no pós-operatório tardio, ocasião em que ambos os buracos maculares se apresentavam clinicamente fechados. Todos os pacientes foram submetidos a exame oftalmológico completo e exames complementares, incluindo biomicroscopia do segmento posterior, retinografia e fluoresceinografia. Procedeu-se à avaliação do perfil (corte óptico) e da espessura retiniana pelo RTA em todos os olhos para uma análise comparativa com os exames precedentes. Adotou-se como referência normal na análise da espessura retiniana pelo RTA valores previamente determinados e publicados por nós (Tabela 1$)^{(11)}$. Todos os exames foram analisados por pelo menos dois especialistas em retina e vítreo (M.B.N. e C.B.S.).

\begin{tabular}{|lc|}
\hline \multicolumn{2}{|c|}{$\begin{array}{c}\text { Tabela 1. Espessura retiniana no pólo posterior, medida pelo } \\
\text { RTA, em } \mathbf{3 5} \text { olhos normais }\end{array}$} \\
ESPESSURA & MÉDIA \pm DESVIO PADRÃO $(\boldsymbol{\mu m})$ \\
\hline FOVEAL MÉDIA & $156 \pm 19$ \\
PERIFOVEAL MÉDIA & $191 \pm 26$ \\
PERIFOVEAL MÍNIMA & $150 \pm 24$ \\
PERIFOVEAL MÁXIMA & $232 \pm 38$ \\
\hline
\end{tabular}

\section{Análise da Espessura Retiniana}

Neste estudo foi utilizado o Analisador de Espessura Retiniana (RTA) comercializado pela Talia Technology, Mesaveret Zion, Israel. A aferição é feita pelo laser verde HélioNeônio (HeNe) de $540 \mathrm{~nm}$ de comprimento de onda. O feixe de luz empregado na varredura tem $2 \mathrm{~mm}$ de comprimento vertical por $10 \mu \mathrm{m}$ de largura e a medida é feita pela captura de sua imagem refletida/dispersada sobre determinado ângulo. Essa imagem da interseção do feixe de laser com a retina irá produzir um corte óptico daquela, que é gravado por uma videocâmera e digitalizado. A análise das imagens é, então, realizada automaticamente por meio de um software, sem necessidade de intervenção do operador do aparelho. A separação entre as reflexões correspondentes às interfaces vitreorretiniana e coriorretiniana é medida e corresponderá à espessura da retina. O RTA realiza uma varredura gerando dez cortes ópticos separados cada um por uma distância de $200 \mu \mathrm{m}$, cobrindo uma área de $2 \times 2 \mathrm{~mm}$ da retina em cerca de 200 a 400 milissegundos. A varredura pode ser realizada em nove áreas (área total $6 \times 6 \mathrm{~mm}$ ), $\mathrm{o}$ que irá corresponder a 900 pontos de medição e irá abranger o mapeamento dos $20^{\circ}$ centrais do pólo posterior ${ }^{(11,13)}$.

Imediatamente após a aquisição das imagens, os dez cortes ópticos são vistos no monitor para apreciação de sua qualidade (Figura 1). Nesta fase é possível detectar alterações existentes no perfil da retina, como ocorre, por exemplo, nos buracos maculares. Há, então, a possibilidade de impressão do laudo padrão apresentado na tela do monitor, seja como

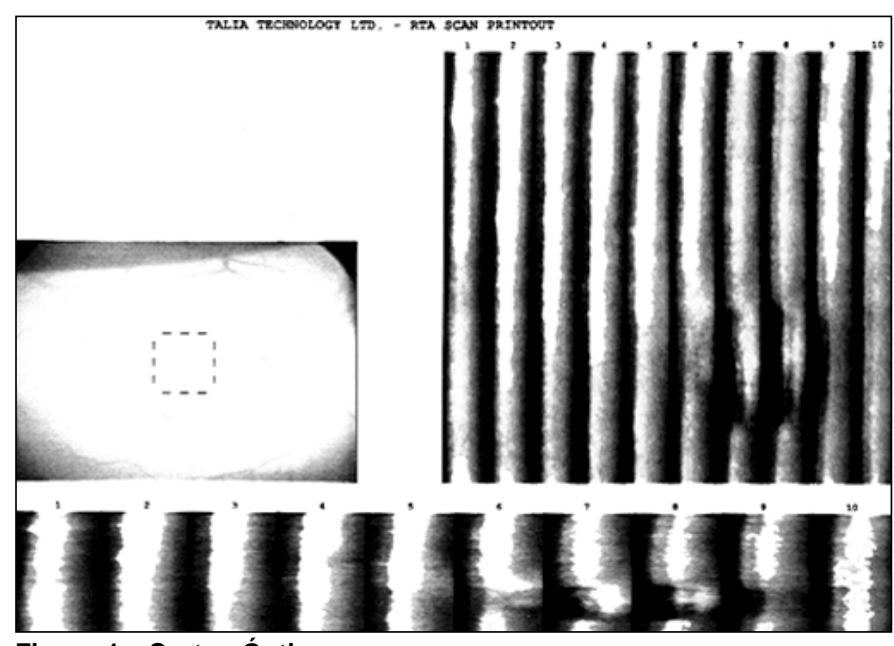

Figura 1 - Cortes Ópticos 
representação bidimensional da espessura retiniana, codificada em cores, ou representação semelhante, porém comparativa (desvio do normal), com valores obtidos de um grupo de 400 pacientes normais (segundo informações do fabricante).

\section{RESULTADOS}

Os principais dados relativos aos pacientes estudados estão na Tabela 2. Dos dez olhos estudados, oito foram examinados antes de qualquer tratamento e dois foram examinados no pós-operatório de cirurgia para o buraco macular, que consistiu de vitrectomia via pars plana e tamponamento interno com gás C3F8. Dos oito olhos não tratados, um apresentava buraco macular estágio $1 \mathrm{~B}$, dois apresentavam estágio 2, dois apresentavam estágio 3 e três olhos apresentavam estágio 4. Os dois olhos operados apresentavam buraco macular clinicamente fechado. No paciente 6 os exames foram realizados nove meses após a cirurgia. Nesse olho o buraco macular estava totalmente selado e apresentava discreta hiperpigmentação central com cerca de $50 \mu \mathrm{m}$ e halo circunjacente de rarefação do epitélio pigmentar da retina. Esse paciente apresentava, no pré-operatório, AV de 20/200 e buraco macular estágio 3 . O fechamento do buraco macular foi acompanhado de melhora da AV para 20/60. No paciente 5 os exames foram realizados dois meses e meio após a cirurgia. No pré-operatório apresentava buraco macular estágio $3 \mathrm{e} \mathrm{AV} \mathrm{de}$ $20 / 200$. No exame realizado após a cirurgia, o buraco macular encontrava-se fechado e a AV era de 20/40. As retinografias colorida e em aneritra documentaram o diagnóstico de buraco macular em todos os olhos.

Dos oito olhos com buraco macular não operados, sete apresentaram hiperfluorescência central correspondente ao buraco macular (Figura 2). Um olho, com buraco macular estágio 1B (paciente 2), mostrou ausência de hiperfluorescência central. O olho esquerdo do paciente 5 (Figura 3 ) e o olho direito do paciente 6 , previamente operados, apresentavam hipofluorescência central, por efeito máscara, devido ao

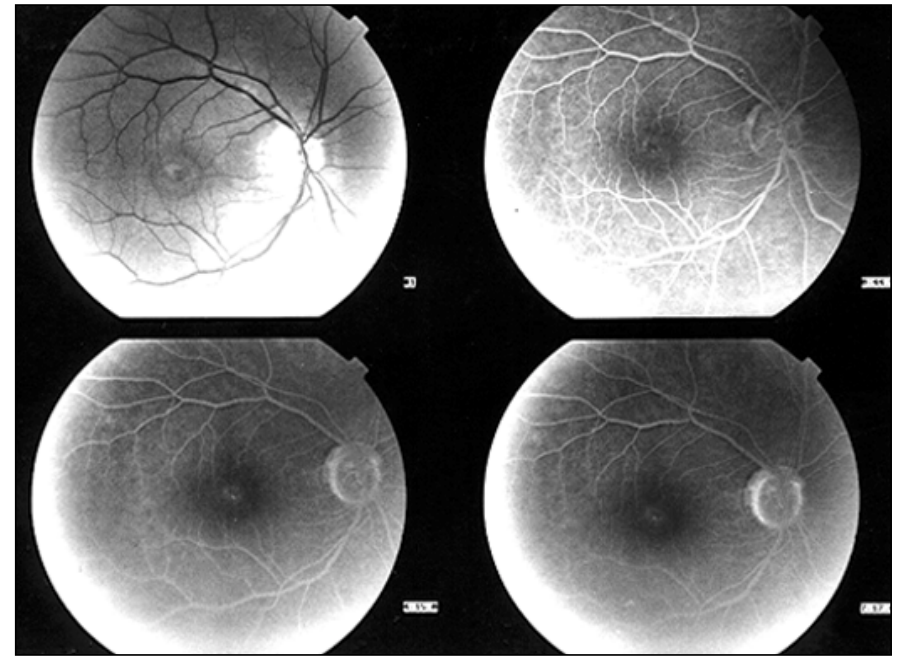

Figura 2 - Hiperfluorescência central em olho com buraco macular

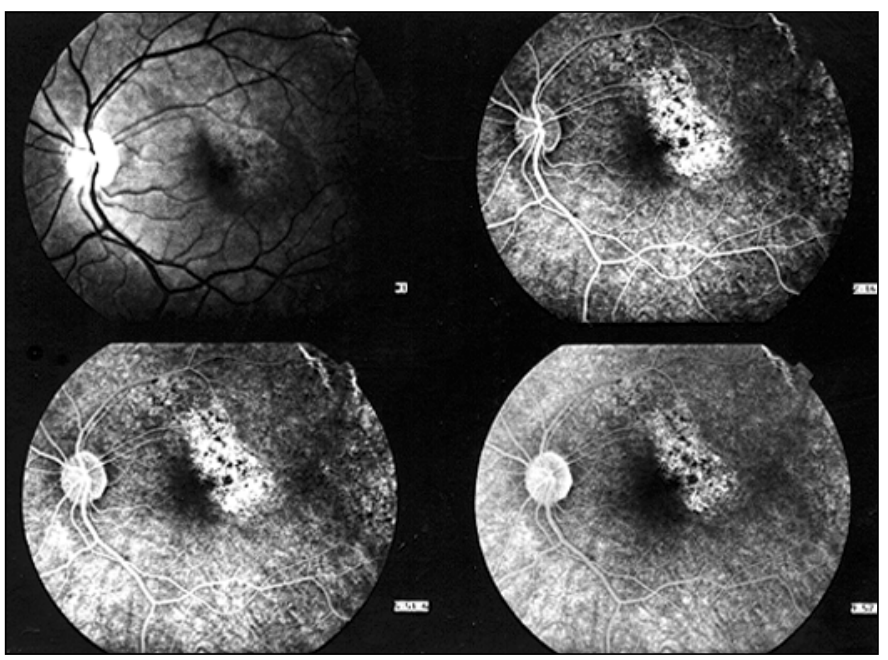

Figura 3 - Fluoresceinografia mostrando buraco macular fechado no pósoperatório. Observa-se hipofluorescência central, por efeito máscara, devido a pigmento na fóvea e hiperfluorescência temporal superior se estendendo até próximo à arcada temporal superior por rarefação do EPR

\begin{tabular}{|c|c|c|c|c|c|c|c|c|c|}
\hline \multirow[t]{2}{*}{ PACIENTE } & \multirow[t]{2}{*}{ OLHO } & \multirow[t]{2}{*}{ AV } & \multirow[t]{2}{*}{ BIO R e V } & \multirow[t]{2}{*}{ RET } & \multirow[t]{2}{*}{ AF } & \multicolumn{4}{|c|}{ RTA } \\
\hline & & & & & & EFM & EPf Média & EPf Mínima & EPf Máxima \\
\hline 1 & $\begin{array}{l}\text { OD } \\
\text { OE }\end{array}$ & $\begin{array}{l}20 / 80 \\
20 / 80\end{array}$ & $\begin{array}{l}\text { BM4 } \\
\text { BM4 }\end{array}$ & $\begin{array}{l}\text { BM } 3 \text { ou } 4 \\
\text { BM } 3 \text { ou } 4\end{array}$ & $\begin{array}{l}\text { HIPER } \\
\text { HIPER }\end{array}$ & $\begin{array}{l}299 \\
233\end{array}$ & $\begin{array}{l}303 \\
161\end{array}$ & $\begin{array}{l}228 \\
123\end{array}$ & $\begin{array}{l}419 \\
200\end{array}$ \\
\hline 2 & $\begin{array}{l}\text { OD } \\
\text { OE }\end{array}$ & $\begin{array}{c}C D \\
20 / 25\end{array}$ & $\begin{array}{c}\text { BM4 } \\
\text { BM 1B }\end{array}$ & $\begin{array}{c}\text { BM } 3 \text { ou } 4 \\
\text { BM 1B }\end{array}$ & $\begin{array}{l}\text { HIPER } \\
\text { NORMAL }\end{array}$ & $\begin{array}{l}333 \\
230\end{array}$ & $\begin{array}{l}326 \\
257\end{array}$ & $\begin{array}{l}230 \\
185\end{array}$ & $\begin{array}{l}414 \\
339\end{array}$ \\
\hline 3 & $\begin{array}{l}\text { OD } \\
\text { OE }\end{array}$ & $\begin{array}{c}20 / 400 \\
20 / 80\end{array}$ & $\begin{array}{l}\text { BM3 } \\
\text { BM2 }\end{array}$ & $\begin{array}{c}\text { BM } 3 \text { ou } 4 \\
\text { BM2 }\end{array}$ & $\begin{array}{l}\text { HIPER } \\
\text { HIPER }\end{array}$ & $\begin{array}{l}318 \\
344\end{array}$ & $\begin{array}{l}244 \\
222\end{array}$ & $\begin{array}{l}177 \\
187\end{array}$ & $\begin{array}{c}90 \\
265\end{array}$ \\
\hline 4 & $\begin{array}{l}\text { OD } \\
\text { OE }\end{array}$ & $\begin{array}{c}20 / 100 \\
20 / 50\end{array}$ & $\begin{array}{l}\text { BM3 } \\
\text { BM2 }\end{array}$ & $\begin{array}{c}\text { BM } 3 \text { ou } 4 \\
\text { BM2 }\end{array}$ & $\begin{array}{l}\text { HIPER } \\
\text { HIPER }\end{array}$ & $\begin{array}{l}\text { NR } \\
\text { NR }\end{array}$ & $\begin{array}{l}\text { NR } \\
\text { NR }\end{array}$ & $\begin{array}{l}\text { NR } \\
\text { NR }\end{array}$ & $\begin{array}{l}\text { NR } \\
\text { NR }\end{array}$ \\
\hline $5^{*}$ & OE & $20 / 40$ & BM fechado & BM fechado & HIPO & 175 & 171 & 140 & 204 \\
\hline $6^{*}$ & OD & $20 / 60$ & BM fechado & BM fechado & HIPO & 149 & 198 & 171 & 221 \\
\hline \multicolumn{10}{|c|}{$\begin{array}{l}\text { Legenda: OD: Olho Direito; OE: Olho Esquerdo; AV: Acuidade visual; CD: conta dedos; Bio R e V: biomicroscopia do segmento posterior, RET: retinografia, AF: } \\
\text { fluoresceinografia, EFM: espessura foveal média; EPf: espessura perifoveal; BM: Buraco Macular; NR: imagens não registráveis; HIPER: hiperfluorescência; HIPO: } \\
\text { Hipofluorescência central } \\
{ }^{*} \text { Os pacientes } 5 \text { e } 6 \text { foram previamente submetidos a vitrectomia }\end{array}$} \\
\hline
\end{tabular}


pigmento, com hiperfluorescência adjacente, por efeito janela. É possível que essa hiperfluorescência seja devido a fototoxicidade ou a remoção da membrana limitante interna.

Em dois dos dez olhos com buraco macular evidenciado pela biomicroscopia do segmento posterior, retinografia e fluoresceinografia, não foi possível a análise pelo RTA devido à opacidade dos meios (paciente 4). Analisando-se a medida da espessura foveal média por meio do RTA observou-se que nos seis olhos com buraco macular não operado houve aumento da espessura foveal média, da espessura perifoveal média em quatro olhos, da espessura perifoveal mínima em dois olhos e da espessura perifoveal máxima em três olhos (Figura 4). Os dois olhos previamente operados e que mostravam o buraco macular clinicamente fechado apresentaram valores normais em todas as espessuras: espessura foveal média, espessura perifoveal média, espessura perifoveal mínima e espessura perifoveal máxima (Figura 5). Na análise dos cortes ópticos observou-se uma depressão central correspondente à área do buraco macular nos seis olhos não operados.

\section{DISCUSSÃO}

Na avaliação de olhos com buraco macular a biomicroscopia do segmento posterior tem um papel fundamental. Para se obter informações confiáveis com esse exame, requer-se uma considerável experiência do examinador. As informações fornecidas por esse exame, embora extremamente importantes, são subjetivas e preponderantemente qualitativas. Em tese, exames complementares capazes de fornecer informações objetivas e quantitativas, e que não necessitem de grande habilidade para a sua realização e experiência para a sua interpretação, poderiam ser extremamente úteis na avaliação de portadores de buraco macular e na sua diferenciação de pseudoburaco macular. Tais exames poderiam fornecer informações significativas a respeito da evolução e patogênese do buraco macular, bem como na avaliação da eficácia da terapêutica cirúrgica dos buracos maculares e mais precisamente dos achados morfológicos associados com a melhora funcional pós-tratamento. Esses achados poderiam não apenas esclarecer mecanismos da recuperação anatômica e funcional, como também constituir metas a serem obtidas com o tratamento cirúrgico.

O RTA é um novo instrumento idealizado para avaliar, quantitativa e qualitativamente, a topografia e a espessura retiniana no pólo posterior e, dessa forma, permitir um melhor estudo de algumas maculopatias, incluindo o buraco macular.

Estudos prévios demonstraram que o RTA apresenta quatro importantes propriedades: múltiplos cortes ópticos da retina, mapeamento da espessura retiniana, alta reprodutibilidade e um tempo de aquisição de imagens relativamente pequeno, o que minimiza artefatos causados pela movimentação do olho ${ }^{(11-13)}$. O RTA caracteriza-se, ainda, por não ser invasivo e não necessitar de contato com a superfície ocular.

Uma dilatação pupilar de pelo menos $5 \mathrm{~mm}$ é necessária para permitir a obtenção de imagens ${ }^{(11)}$. Essa limitação, embora significativa, é contornável na maioria dos casos. Seria desejável que o aparelho pudesse obter imagens em olhos em que não se consegue uma boa midríase. Outra importante limitação é a sua incapacidade de obter imagens em presença de opacidades significativas dos meios. Essa limitação ficou

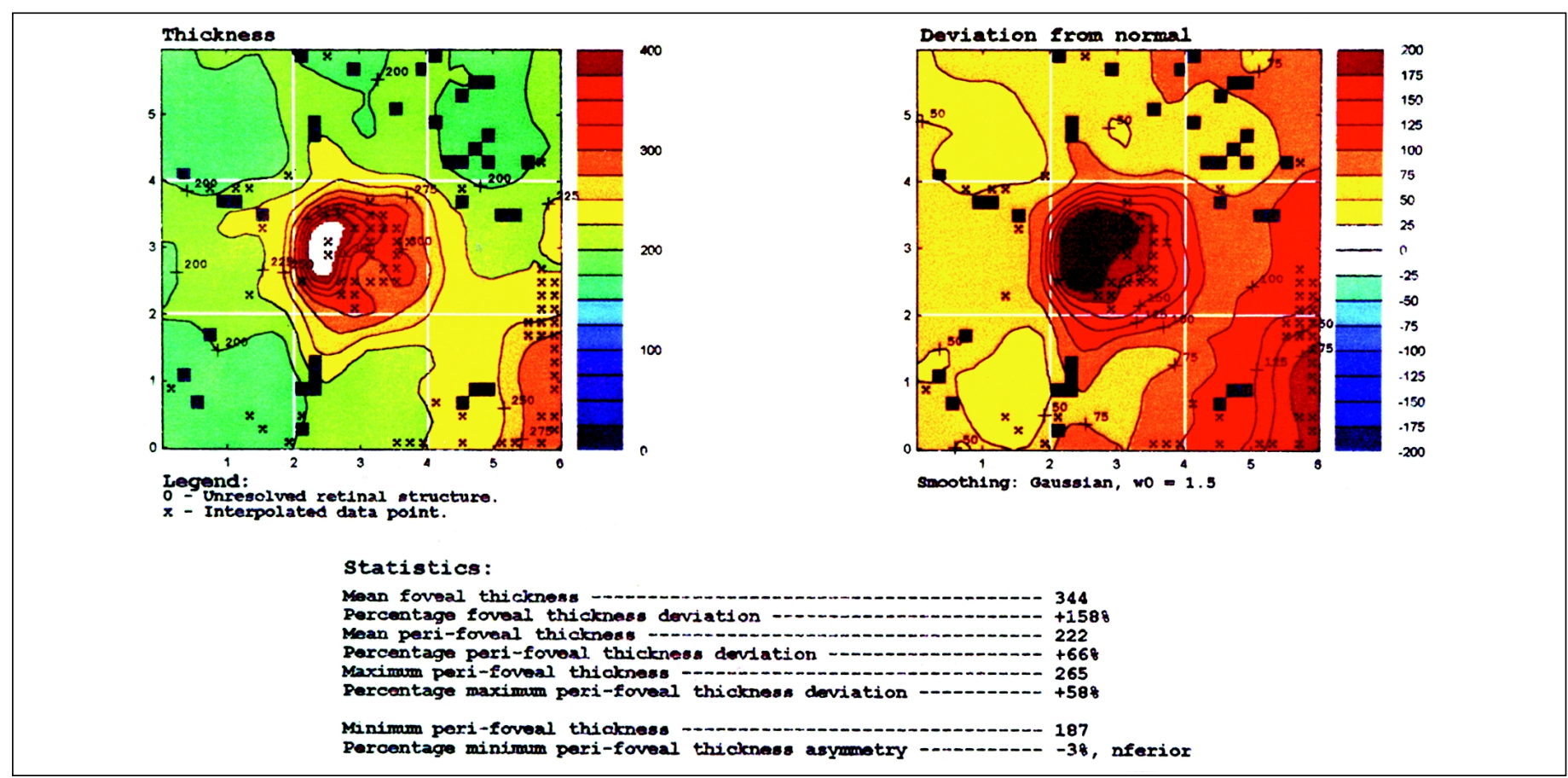

Figura 4 - RTA mostrando aumento da espessura foveal média, no mapa comparativo, em paciente com buraco macular estágio 2 (paciente 3 - OE) 


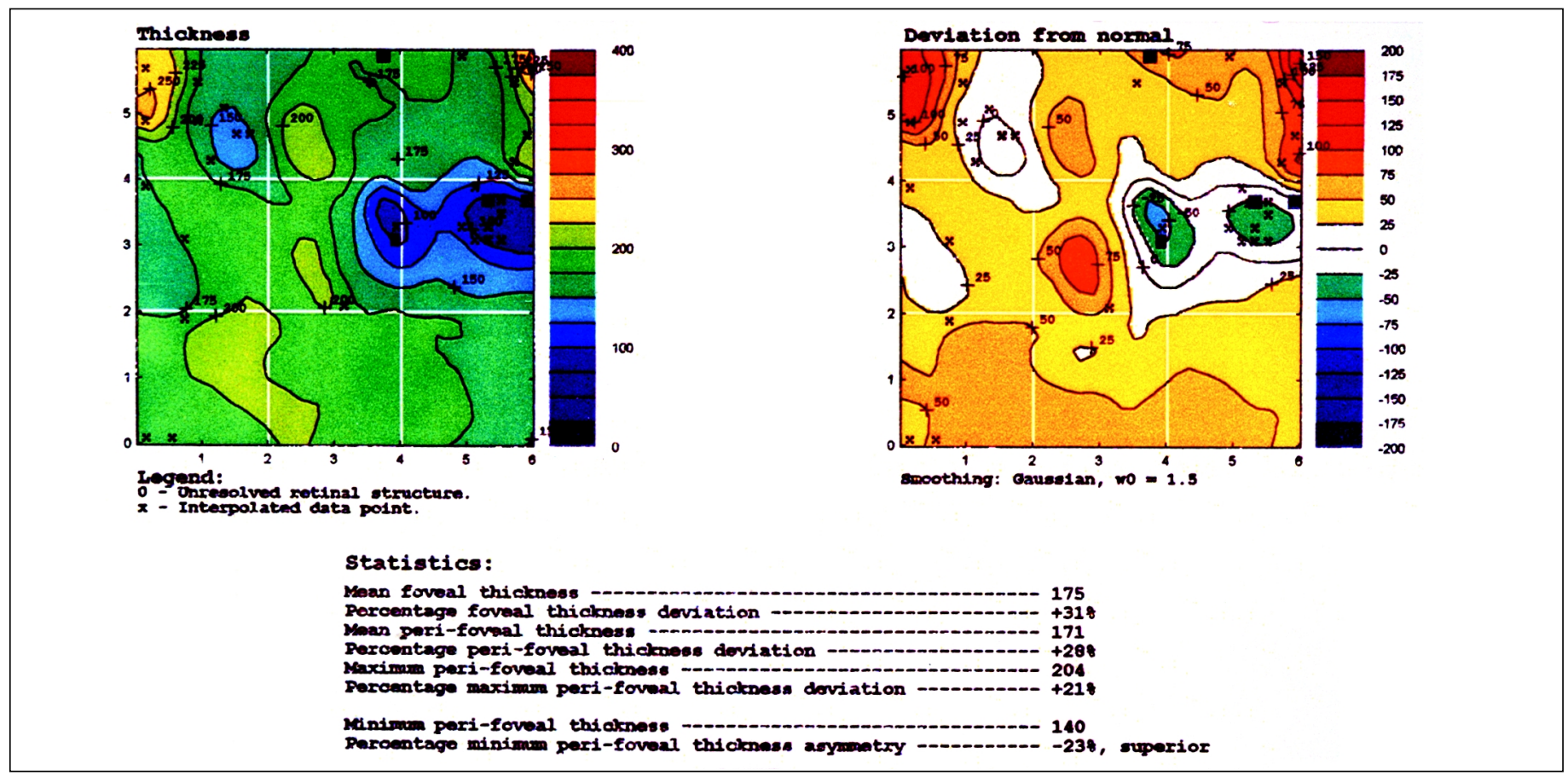

Figura 5 - Mapa comparativo mostrando a espessura foveal média normal em um olho com buraco macular fechado

patente no presente estudo, no qual, em dois dos dez olhos estudados, não foi possível a obtenção de imagens devido à presença de catarata. Deve-se ressaltar que, nos dois olhos, a catarata apresentava densidade de discreta a moderada, porém ainda permitia uma boa avaliação fundoscópica pelos exames convencionais.

A literatura mostra que o RTA pode produzir imagens com características próprias em casos de buraco macular, de membrana epirretiniana, de espessamento da superfície posterior do vítreo e de mudanças nos cistos intra-retinianos, auxiliando, assim, o estabelecimento do diagnóstico diferencial do buraco macular ${ }^{(5,12)}$. Asrani et al. $(1997)^{(5)}$ determinaram que em $42 \%$ dos casos com suspeita ou diagnóstico clínico de buraco macular o RTA mostrou resultados discordantes do exame clínico. Esse achado demonstra que o diagnóstico clínico requer experiência considerável para estabelecer um diagnóstico preciso. O RTA poderia, assim, proporcionar maior segurança em casos com diagnóstico duvidoso. No presente estudo, houve concordância em $100 \%$ dos casos, nos resultados do RTA, utilizando o corte óptico. É importante ressaltar que neste trabalho foram incluídos apenas olhos com buraco macular que não apresentavam dificuldade para o diagnóstico clínico. Esse trabalho não objetivou estudar olhos com pseudoburaco macular, que poderiam ser confundidos com os buracos maculares verdadeiros.

Em olhos com buraco macular existe uma redução da espessura retiniana na região foveal. Neste estudo, entretanto, utilizando-se a análise dos mapas comparativos, não se observou redução da espessura foveal média, como seria esperado, em nenhum dos seis olhos com buraco macular não operado. Observou-se, pelo contrário, aumento da espessura foveal média em todos os seis olhos. Que tenhamos conhecimento, este é o primeiro relato da literatura que mostra aumento da espessura foveal, pelo mapa comparativo do RTA. Acreditamos que esse fato seja decorrente da extrema dificuldade de fixação dos olhos portadores dessa patologia durante a realização do exame. Outra explicação é inerente a limitações próprias do aparelho. Os mapas topográficos são realizados pelo programa do computador a partir da identificação de duas interfaces, vítreo-retina e retina-epitélio pigmentar da retina, segundo informações do próprio fabricante. A ausência de uma dessas interfaces determina falhas no programa, o que pode explicar esse aumento da espessura foveal medida pelo mapa topográfico, em olhos com buraco macular.

As séries histopatológicas mais recentes de buraco macular demonstram edema intra-retiniano microcístico circundando o buraco macular em $79 \%$ dos casos ${ }^{(8)}$. Esse fator pode explicar o aumento da espessura da retina perifoveal, medido pelo RTA. Estudos adicionais são necessários para se analisar se cortes topográficos com as espessuras (foveal média, perifoveal média, perifoveal mínima e perifoveal máxima) reproduzem-se consistentemente em casos de buraco macular, se existe de fato um eventual aumento da espessura retiniana na mácula - não detectável pelo exame clínico mas detectável pelo RTA- ou se esse aumento é decorrente de artefato e em quais circunstâncias esse artefato é produzido. Apesar dessas observações, os estudos em corte óptico fornecem informações importantes a respeito da existência ou não de buraco macular e medidas precisas da sua profundidade, bem como informações sobre a sua conformação. 
É interessante notar que os dois olhos previamente operados apresentaram buraco macular fechado, melhora de acuidade visual e espessura foveal média e espessura perifoveal média normais. Esses achados sugerem que a melhora anatômica (e da acuidade visual) se acompanha de normalização da espessura retiniana medida pelo RTA. De certa forma, esse resultado é condizente com a reaplicação do descolamento de retina sensorial, obtida com a vitrectomia e com o tamponamento interno com gás, reaplicação esta que justifica a melhora anatômica e funcional observada nesses olhos.

Em síntese, o presente trabalho mostra os achados do RTA próprios de olhos com buraco macular, porém estudos envolvendo um número maior de casos são necessários para estabelecer os achados característicos do buraco macular em seus diferentes estágios.

\section{ABSTRACT}

Objective: To evaluate the Retinal Thickness Analyzer (RTA) findings in eyes with macular hole and compare them to findings of retina and vitreous biomicroscopy, retinography and fluorescein angiography. Methods: The authors studied ten eyes of six patients with the diagnosis of macular hole, using retinal thickness analyzer, retina and vitreous biomicroscopy, retinography and fluorescein angiography. Eight eyes had been examined before any type of treatment, and two, after treatment for macular hole by vitrectomy. Results: In two eyes with the diagnosis of macular hole by clinical examination and other complementary examinations, the media opacities prevented retinal thickness analyzer analysis. The retinal thickness analyzer profile map (optical cut) showed agreement with the clinical findings and the findings by other examinations performed in the eight eyes where they were possible. The topographic map of the two eyes that presented a closed macular hole after surgery, showed normal mean foveal thickness and in the six eyes with macular hole the mean foveal thickness was increased. Conclusion: The retinal thickness analyzer profile map showed evidence of macular hole, gave information about width and depth and proved the closing of the macular hole after surgery. The retinal thickness analyzer topographic map showed normal mean foveal thickness in the two cases of closed macular hole and, surprisingly, increased mean foveal thickness in all six cases with open holes.

Keywords: Retinal tickness analyzer; Ophthalmological diagnostic techniques; Retinal perforations

\section{REFERÊNCIAS}

1. Veiga-Reis F, Dias RB, Nehemy MB. Buraco macular: diagnóstico diferencial Teste Watzke-Allen e teste da mira do laser. Arq Bras Oftalmol 1997;60:631-4.

2. Dantas, AM. Doenças da Retina. Rio de Janeiro: Cultura Médica, 1989.

3. Vale MB, Veiga-Reis F, Nehemy MB. Correlação clínico-ecográfica nos buracos maculares idiopáticos. Rev Bras Oftalmol 1998; 57:33-7.

4. Gass, JDM. Stereoscopic atlas of macular diseases: diagnosis and treatment, $3^{\text {rd }}$ ed. St. Louis: CV Mosby, 1987. p. 684-93.

5. Asrani S, Zeimer R, Goldberg MF, Zou S. Serial optical sectioning of macular holes at different stages of development. Ophthalmology 1998;105:66-77.

6. Morgan CM., Schatz H. Involutional macular thinning. A pre-macular hole condition. Ophthalmology 1986;93:153-61.

7. McDonnell PJ, Fine SL, Hills AJ. Clinical features of idiopathic macular cysts and holes. Am J Ophthalmol 1982;93:777-86.

8. Guyer DR, Green WC, De Bustros S, Fine SL. Histopathologic features of idiopathic macular holes and cysts. Ophthalmology 1990;97:1045-51.

9. Kornzweig AL, Feldstein M. Studies of the eye in old age hole in the macula. A clinico-pathologic study. Am J Ophthalmol 1949;243-7.

10. Yasukawa T, Kiryu J, Tsujikawa A, Dong J, Suzuma, Takagi H, et al. Quantitative analysis of foveal retinal thickness in diabetic retinopathy with the scanning retinal thickness analyzer. Retina 1998;18:150-5.

11. Zisman M, Nehemy MB, Aguiar AP, Cunha AS, Siqueira CB. Estudo da espessura foveal e perifoveal em indivíduos normais por meio do analisador de espessura retiniana. Rev Bras Oftalmol 1998;57:929-33.

12. Asrani S, Zeimer R, Goldberg MF, Zou S. Application of rapid scanning retinal thickness analysis in retinal diseases. Ophthalmology 1997;104: 1145-51.

13. Zeimer R, Shahidi M, Mori M, Zou S, Asrani S. A new method for rapid mapping of the retinal thickness at the posterior pole. Invest Ophthalmol Vis Sci 1996;37:1994-2001.

\section{Novidades na Internet!!!}

Agora no site CBO você tem disponível todas as informações na íntegra dos Arquivos Brasileiros de Oftalmologia http://www.cbo.com.br/abo 